\title{
REVISIONES
}

\section{DESAFIOS A LA FORMACION DOCENTE: INCLUSION EDUCATIVA*}

\author{
Challenges to teacher education: Educational inclusion
}

\author{
Marta Infante \\ Pontificia Universidad Católica de Chile, Facultad de Educación, Av. Vicuña Mackenna 4860, Macul, \\ Santiago, Chile, minfantj@uc.cl
}

\section{Resumen}

El presente artículo busca cuestionar la inclusión educativa, entendida como un proceso que intenta abordar situaciones y espacios de exclusión en el sistema educativo y los desafíos que éste plantea a la formación de profesores. Primero, se analiza el concepto de inclusión desde una perspectiva contemporánea; segundo, se discuten los riesgos que tiene el entenderla desde el campo de la educación especial; tercero, se plantean algunas limitaciones a la inclusión educativa existentes en la formación docente, y cuarto, se describen tres posibilidades a considerar para la formación de un nuevo profesional que utilice la inclusión educativa como una forma de responder, abordar y celebrar la diversidad en nuestro sistema educativo.

Palabras clave: inclusión educativa, formación inicial docente, exclusión, educación especial, identidades, aprendizaje.

\begin{abstract}
This article aims to analyze educational inclusion as an educational device that breaks with exclusionary practices and spaces in the educational system and the challenges that offer to pre-service teachers' formation. First, the concept of inclusion from a contemporary perspective is examined, second, the risks of understanding it from the special education field are discussed, third, current restrictions to inclusion development in teachers' formation programs are shown and fourth, three proposals for a new teacher that utilizes inclusion as a road to respond, accept, and celebrate diversity in our educational system are described.
\end{abstract}

Key words: educational inclusion, teachers' initial formation, exclusion, special education, identities, learning.

\footnotetext{
* $\quad$ Este artículo forma parte del Proyecto FONDECYT Nº 1070802.
} 


\section{INCLUSION EDUCATIVA: AMPLIANDO O RESTRINGIENDO MIRADAS}

El concepto de inclusión ha adquirido un énfasis especial durante los últimos años en el contexto educativo latinoamericano y particularmente el chileno, visibilizándose en ámbitos como políticas públicas y acciones gubernamentales. Esta representación del concepto de inclusión regula no solo las prácticas educacionales (enseñanza, metodología, currículum, entre otras) sino las ideas sobre situaciones de exclusión, diversidad y de manera significativa, sobre la construcción de identidades. Este último aspecto hace referencia a la construcción de sujetos/estudiantes de pedagogía desde discursos de inclusión que circulan y se reproducen en el ámbito educacional. En este sentido, el significado de inclusión tiene implicancias en el estudiante que se construye (Hall, 1997).

Históricamente la inclusión educativa como concepto y práctica en contextos escolares comienza a principios de los 80 en los Estados Unidos y en Europa, como una iniciativa focalizada hacia los estudiantes con discapacidad (Fuchs y Fuchs, 1994; Lipsky y Gartner, 1996). Sin embargo, durante las últimas décadas, ese foco ha cambiado y se plantea como un nuevo desafío: hacer las prácticas inclusivas en educación accesibles a todas las personas. Lo anterior implicaría un cuestionamiento de los supuestos normativos existentes sobre el aprendizaje y la enseñanza de manera de responder a la diversidad de los sujetos implicados en el contexto educativo en cuanto a raza, etnicidad, lenguaje, género, nacionalidad, entre otras diferencias que van más allá de la habilidad para aprender. Este último planteamiento refuerza la idea de que la inclusión educativa se constituye en una forma de responder y abordar la diversidad en contextos educacionales. Se amplía así la representación de quiénes son los sujetos interpelados en y por esta nueva significación, abriendo espacio a nuevas subjetividades y a las intersecciones de sus marcadores (ej., discapacidad, etnia, género, entre otras). El concepto de subjetividades es utilizado para complejizar la construcción de sujeto desde una perspectiva más contemporánea donde se hace referencia a la idea de construcción dinámica. Así, los sujetos tienen la posibilidad de habitar infinitas formas de ser sujeto y resistir a dispositivos normalizadores que lo limitan desde identidades movibles y transitorias (Rose, 1999).

La migración del concepto de inclusión desde la educación especial a espacios relacionados con la educación general (o regular) ha producido avances en la comprensión del fenómeno educativo contemporáneo al abordar la variabilidad que existe en el aprendizaje de los estudiantes. En Chile, por ejemplo, las salas de clase de escuelas regulares han incrementado la cantidad de estudiantes con discapacidad y estudiantes extranjeros desafiando las metodologías tradicionales de enseñanza destinadas a un estudiante que no tiene discapacidad y que ha nacido en este país. Sin embargo, el concepto de inclusión también es representado como un equivalente al de asimilación debido a que sus orígenes están epistemológicamente ligados a una tradición positivista, propia de la educación especial tradicional. Slee (2001) refuerza esta idea al argumentar la existencia de representaciones limitadas sobre la educación inclusiva, entre las que se cuentan una constricción a un marco tradicional de la educación especial sustentada en una visión médica de la diferencia y una desconexión de las políticas de inclusión educativa del contexto general de políticas educacionales.

Desde otra perspectiva, organismos internacionales como la UNESCO (2005) han explicitado la idea de que la inclusión educativa debiera sustentarse en los derechos 
humanos, donde el acceso y participación a una educación de calidad es un imperativo. En este sentido, todos los seres humanos, independiente de su etnia, género, forma de aprender, etc., deberían gozar y ejercer el derecho a la educación.

El movimiento inclusivo en educación, cualquiera sea su comprensión, ha movilizado la construcción de una serie de políticas a nivel de organismos internacionales que han sido traducidas en acciones educacionales referidas fundamentalmente a permitir que todos los niños tengan acceso al sistema educacional regular. Ejemplos de esto en Chile son la construcción de una política de educación especial, el desarrollo de un programa intercultural y la construcción de proyectos de asistencia técnica a escuelas que evidencian bajos logros en mediciones de aprendizaje y altas tasas de repitencia y deserción escolar, entre otros (MINEDUC, 2005, 2007). Así, estas políticas han posibilitado la creación de programas compensatorios en la escuela para que aquellos niños que habían sido excluidos y marginados del sistema pudiesen participar de él. Sin embargo, la inclusión educativa a pesar de que se ha convertido en un concepto de uso bastante amplio en el contexto educativo, trae consigo diversos discursos no siempre convergentes en cuanto a su significado y comprensión. Al respecto, Graham (2006: 4) argumenta que el término inclusión:

...sugiere un 'traer dentro'; un término que presupone un todo en el que algo o alguien puede ser incorporado...Sería razonable argumentar que hay un centro implícito al término inclusión, para el que se privilegian nociones discursivas de lo preexistente por medio de incluir al otro en un espacio prefabricado, naturalizado.

Desde esta perspectiva, se esperaría que el término implícitamente denotara un espacio que está conformado por aquellos sujetos que sí han podido ser parte de un sistema escolar regular o normal. Aquellos niños que aprenden como el promedio y que tradicionalmente han formado parte de lo que se denomina escuela. Así, el concepto de inclusión hace referencia a la construcción de un 'Otro' (alumno que sale de los límites de la norma), a uno que no ha tenido el privilegio o, en palabras actuales, el derecho de estar en esos espacios educativos. Se busca, por tanto, aproximar a esos Otros a estos espacios regulares construidos social y culturalmente como centros. El desarrollo de espacios particulares como aulas de recursos, adaptaciones al currículum, entre otros, buscan acercar a estos alumnos al desempeño promedio de los alumnos que habitan este centro. Se inicia un círculo a través de procedimientos de diagnóstico donde se pretende visualizar y destacar la distancia que tiene este sujeto de inclusión con respecto al alumno definido como promedio. Mutua y Smith (2006: 126) refuerzan esta premisa al plantear que una gran mayoría de educadores en ejercicio y en formación “....siguen el proceso de diagnóstico y tratamiento del modelo médico, creyendo que asignando una categoría a los estudiantes es el primer paso necesario para proporcionar servicios apropiados a esos estudiantes". En el caso de Chile, este promedio al que se aspira corresponde a un estudiante con un buen rendimiento académico acorde a los estándares educacionales chilenos (currículum escolar), nivel socioeconómico medio-alto, y nacido dentro de los límites geográficos que determinan el país. En relación a esta idea restringida de inclusión, Graham y Slee (2005: 6) plantean que:

Nociones limitadas y modelos de inclusión, como aquellos realizados a través de mecanismos que aseguran la objetivación de la diferencia individual, no sólo resultan en una más compleja 
e insidiosa exclusión pero posiblemente funcionan en refinar la escuela como un campo de aplicación para el poder disciplinario.

Profundizando en la idea de Graham y Slee, la objetivación de la diferencia permite abordar la inclusión desde los alumnos que eran sujetos de prácticas de exclusión y oscurece el centro, dejándolo libre de cuestionamiento. Así el concepto de estudiante promedio continúa fuera de análisis y se constituye en un punto de partida y una meta a alcanzar a través de las prácticas educacionales, configurándose en un artefacto de poder que restringe las prácticas inclusivas más contemporáneas.

Una forma de comprender la inclusión es en relación a sus antecesores en los sistemas educativos latinoamericanos, entre los que se cuenta Chile. Específicamente, la inclusión educativa tiende a relacionarse discursivamente con el concepto de educación especial y por tanto, de integración escolar. Esta última es definida como un conjunto de herramientas educacionales que responden a la educación de estudiantes con necesidades educativas especiales derivadas de discapacidad o con trastornos específicos del lenguaje en un contexto de educación regular. Las herramientas se centran principalmente en apoyos pedagógicos por parte de especialistas (fonoaudiólogos, terapeutas ocupacionales, profesores diferenciales, entre otros) (MINEDUC, 2005).

Esta comprensión de la inclusión ha permitido la evolución de algunas temáticas educacionales y en especial de aquellas relacionadas con la discapacidad. Sin embargo, al relacionarse con este espacio de conocimiento (discapacidad), ha traído ciertas problemáticas producto de la tradición positivista que presenta la educación especial en términos históricos, arrastrando así discursos que entorpecen su (re)configuración (Baker, 2002).

Existen evidencias suficientes para pensar que la presencia de un modelo tradicional de educación especial que sustente las prácticas inclusivas no permite avanzar en un concepto de inclusión más contemporáneo, produciendo así un maquillaje en la escuela donde las prácticas de exclusión se han trasladado a su interior (Slee, 2001). Un ejemplo de lo anterior se observa en la Política Nacional de Educación Especial Chilena (MINEDUC, 2005) cuyo objetivo es compensar la exclusión que tenían algunos estudiantes del sistema de educación regular por presentar una discapacidad. Es así como la traducción de este artefacto social denominado política de educación especial ha permitido que aquellos estudiantes que aprenden de manera distinta a la mayoría puedan participar del sistema educativo común, teniendo acceso a una educación regular. Sin embargo, ha legitimado nuevas prácticas de exclusión al interior de la escuela. Específicamente, aparece la imperiosa necesidad de establecer categorías diagnósticas precisas para aquellos estudiantes, antes segregados de las escuelas regulares, con el propósito de fijar subvenciones económicas especiales y distintas al resto de los estudiantes. Con esta asignación categórica de quién es ese sujeto especial se inicia un círculo regulatorio que más que abrir espacios de participación, los restringe. Dependiendo de cuanto se aleje el desempeño académico de este sujeto especial con respecto al currículum regular chileno, será el tiempo que podrá compartir con sus compañeros en el aula regular (MINEDUC, 2005). En el caso de que esta distancia sea significativa (mejor aún si es con carácter estadístico), deberá habitar en un nuevo espacio, denominado aula de recursos. De esta forma, en el sistema educacional chileno actual se configuran cuatro opciones de participación para los sujetos con discapacidad, entre las que se cuentan: a) Asistencia a todas las actividades del curso común y atención complementaria de profesionales especialistas en el aula 
de recursos, b) asistencia a todas las actividades del curso común, excepto en aquellas áreas en las que requiera de mayor apoyo, las que deberán ser realizadas en el aula de recursos, c) asistencia en la misma proporción de tiempo al aula de recursos y al aula común y d) asistencia a todas las actividades en el aula de recursos y participación con los alumnos del establecimiento común, en recreos, actos o ceremonias oficiales del establecimiento o de la localidad, y actividades extraescolares en general.

En relación a estas opciones de participación que tendría el sujeto a incluir, Erevelles (2006) describe el concepto de prótesis desarrollado por estos estudiantes como mecanismo que les permite acercarse al rendimiento y conducta normal esperada en la escuela y por tanto, a los espacios del currículum que tienen los demás estudiantes. Ella argumenta en relación a la construcción de este nuevo artefacto educativo lo siguiente:

...en la mayoría de los contextos educacionales, los estudiantes identificados como diferentes de la norma por su raza, clase, género, etnia y/o orientación sexual son validados si y solo si pueden demostrar prácticas de prótesis que les posibilitan pasar como no verdaderamente diferentes de la norma por medio de ocultar su dis/capacidad. Como resultado, la discapacidad se vuelve en un puente discursivo que simultáneamente explica y expone la construcción social de la diferencia en educación junto con los ejes de raza, clase, género, etnia y/o orientación sexual (Everelles, 2006: 368).

Lo anterior también se puede visualizar en el ámbito de las políticas interculturales. A modo de ejemplo, en Chile se instaura un sistema de educación intercultural bilingüe con el propósito de permitir a estudiantes indígenas (Mapuche, Aymara, Rapa Nui y Quechua) desenvolverse adecuadamente tanto en su cultura de origen como en la sociedad global. Específicamente, durante el 2006 se aprueba el diseño del subsector de Lengua Indígena que busca garantizar la enseñanza de las lenguas indígenas en aquellas unidades educativas con presencia mayoritaria de niñas y niños de ascendencia indígena (MINEDUC, 2007). Se refuerza así en Chile, al igual que en otras comunidades latinoamericanas, la necesidad de desarrollar las lenguas indígenas y aumentar el grado de participación de las comunidades locales en los programas educativos. Sin embargo, a diferencia de otros países, la mayoría de los grupos indígenas en Chile no hablan su lengua nativa. Un estudio realizado por Irarrázabal y Morandé (2007) muestra que cerca del $80 \%$ de sujetos que se definen como mapuches no habla su propia lengua (Mapudungún) y mayoritariamente se encuentran en los estratos socioeconómicos bajos. Lo anterior muestra cómo se desarrollan políticas educativas para grupos específicos que históricamente habían sido excluidos del sistema educacional regular, como por ejemplo discapacidad y etnia. Retomando el concepto de prótesis de Erevelles (2006), se puede visualizar en el área de la interculturalidad que aquellos niños que aprendan su lengua nativa, sin importar si es significativo para ellos, su familia o su comunidad, serán exitosos a nivel académico. Por lo tanto, el aprendizaje de una nueva lengua permitiría a los estudiantes acercarse a un concepto de indígena esperado social y educacionalmente.

Lo anteriormente expuesto muestra algunos ejemplos de cómo discursivamente se ha ampliado el concepto de inclusión a gran parte del sistema educativo, incrementando así la construcción de políticas educativas focalizadas hacia estudiantes con alguna discapacidad o que provienen de grupos culturales distintos al mayoritario y que tradicionalmente habían sido excluidos del sistema educacional regular. Además, esta ampliación tiene una base epistémica circunscrita marcadamente a un modelo médico de considerar la 
diferencia, lo que sin duda trae conflictos a los sistemas educacionales y a los sujetos que participan en ellos.

Hasta aquí, se ha analizado el tema de la inclusión desde la mirada de los estudiantes. Sin embargo, los sujetos 'a incluir' no sólo son los que reciben beneficios (acceso a una escuela regular) y limitaciones (acceso a nuevos espacios de marginación) de las prácticas inclusivas, sino también aquellos sujetos que deben facilitar este proceso: las profesoras y los profesores del sistema educativo. Es en este contexto que las nuevas prácticas inclusivas no sólo se (re)producen en el contexto escolar sino en un ámbito educativo mayor: los centros de formación de profesores, planteando así un desafío a estas instituciones y a los nuevos profesores en formación.

\section{FORMACION DE PROFESORES: RIESGOS Y POSIBILIDADES}

Los centros de educación superior también se han visto afectados por las variaciones y múltiples significados que ha tenido el concepto de inclusión educativa. La construcción de políticas compensatorias en educación ha presionado fuertemente la formación de profesionales que sean capaces de reconocer y valorar la diversidad, de modo de promover comunidades educativas inclusivas.

Las teorías contemporáneas del aprendizaje plantean que los profesores y las profesoras deben ser personas capaces de enseñar en contextos diversos de aprendizaje (Ainscow, 2001; Beyer, 2001; Riehl, 2000). Lo anterior es altamente relevante dado que datos provenientes de estudios chilenos muestran la variabilidad del alumnado destacando que el número de estudiantes con necesidades educativas especiales, con ascendencia indígena y con nacionalidad distinta a la chilena ha ido en aumento (MINEDUC, 2007). Sin embargo, gran parte del currículum de educación superior incluye herramientas específicas a nivel de metodologías y didácticas que se centran en el aprendizaje de un escolar promedio (Infante y Matus, 2009). Como resultado, la escuela se proyecta como un espacio homogéneo en cuanto a acciones y sujetos, alejándose significativamente de las realidades de nuestro sistema educacional y con un énfasis implícito en ese centro normativo. Este profesor o profesora en formación va construyendo su identidad profesional en base a capacidades dirigidas a una supuesta normalidad que distan de la escuela a la que se verá enfrentado o enfrentada, produciendo una serie de problemáticas que no es capaz de solucionar una vez que se encuentra en ejercicio (Tenorio, 2007). Del mismo modo, las competencias que definen a un profesor o profesora de aula, delimitadas en su currículum de pregrado, no necesariamente son aquellas que el mismo estudiante en formación ha elaborado a través de sus experiencias en los establecimientos educacionales (Infante, Ortega, Rodríguez, Fonseca, Matus y Ramírez, 2008).

El planteamiento anterior no sólo se evidencia dentro de los programas de formación de docentes de educación general básica y de párvulos, sino que se observa una situación similar en la formación de especialistas que trabajan en el ámbito de la educación (ej., fonoaudiólogos, profesores diferenciales, psicólogos). Los especialistas reciben en su formación una serie de herramientas particulares que les permite apoyar el aprendizaje de grupos minoritarios como alumnos con discapacidad. Paralelamente, en el sistema educacional se observa que producto de políticas educativas con carácter de inclusivas, este especialista debe desplazarse desde la escuela especial a una escuela regular donde 
imperativamente tiene que relacionarse con el resto de los actores educativos, entre ellos los alumnos y alumnas con resultados educativos dentro de la norma y los profesores y las profesoras de aula regular, situación para la que no se encuentra capacitado (Infante, Ortega, Rodríguez, Fonseca, Matus y Ramírez, 2008 y Tenorio, 2007).

En los últimos años las políticas educativas chilenas han hecho demandas concretas a los centros de formación docente. Así, se han introducido temas relacionados con conceptos como necesidades educativas especiales (nee), diversidad, interculturalidad, integración e inclusión, entre otros, a las mallas curriculares de los programas de formación de profesores para la educación básica y parvularia y temas de evaluación y currículum a las mallas curriculares de carreras de especialidad. El objetivo principal es el de proporcionar a los futuros profesores las herramientas para trabajar con adaptaciones al currículum común de la educación escolar chilena. Este aspecto es determinante ya que otorga una intencionalidad clara al contenido que debe estar presente en la formación de profesores. Contenido entendido como conocimiento de los planes y programas que establece la educación chilena y de ciertas modificaciones que deben realizar a estos instrumentos (denominadas adaptaciones curriculares). En este sentido, el currículum universitario se debe centrar en la adquisición de competencias que permitan hacer adaptaciones a sus prácticas pedagógicas y al currículum general. Sin embargo, estas modificaciones realizadas en la educación superior no han sido acompañadas de cambios profundos en el concepto de inclusión que permitan transformar las prácticas educativas de modo de no reproducir acciones de exclusión y segregación en aquellos que históricamente han sido marginados de un sistema de educación regular.

Si la agenda educativa nacional de cualquier país pretende favorecer el desarrollo de una educación inclusiva, la formación de profesionales de la educación es uno de los componentes primordiales. En este sentido, experiencias internacionales (Ainscow, 2005; Slee, 2001) y estudios nacionales (Infante y Matus, 2009; Infante, Ortega, Rodríguez, Fonseca, Matus y Ramírez, 2008 y Tenorio, 2007) permiten visualizar la necesidad de abordar algunos aspectos en esta formación.

El primero consiste en la necesidad de abrir espacios de discusión teórica con una mirada crítica a los cursos de formación docente. Al respecto Slee (2001) cuando hace referencia a las prácticas inclusivas destaca la necesidad de que los futuros docentes y los formadores de estos tengan la posibilidad de comprender sus propias nociones de inclusión para construir espacios de posibilidades distintos que no refuercen las debilidades tradicionalmente visibilizadas de los grupos minoritarios en la escuela. De este modo, un primer paso en la transformación de la formación consistiría en abrir espacios en el currículum universitario de carreras relacionadas con la pedagogía que permitan reflexionar sobre cuál es la construcción de diversidad e inclusión que cada sujeto elabora, con anterioridad al desarrollo de técnicas y herramientas pragmáticas de trabajo (metodologías de enseñanza, evaluación, etc.). Por ejemplo, si consideramos que el diagnóstico y la categorización son la actual puerta de entrada para algunas minorías (ej., étnicas, con discapacidad, entre otras) a la mayoría de las escuelas regulares, este sujeto en formación requeriría desarrollar un sentido crítico de los mensajes implícitos con los que estas categorías son inscritas y una conciencia sobre cómo funcionan en la construcción de regímenes de verdad (sobre, por ejemplo, las habilidades de los estudiantes). Ainscow (2005) refuerza esta idea al explicitar que uno de los aspectos importantes a cuidar en una escuela que busca como premisa la inclusión son los su- 
puestos de déficit que existen en los docentes. Esto debido a que estos supuestos pueden afectar las percepciones que tienen los docentes sobre los estudiantes. Al respecto, Hall (1997: 3) destaca que “... son los participantes en una cultura los que le dan significado a las personas, objetos y eventos...Es por nuestro propio uso de las cosas y por lo que decimos, pensamos y sentimos sobre ellos -como los representamos- que les damos un significado". Claramente, la selección de metodologías y estrategias de enseñanza se construyen a partir de la comprensión y significación que tienen los docentes sobre el aprendizaje y los aprendices (Rice, 2006; Tenorio, 2007).

El segundo aspecto se relaciona con la necesidad de complejizar la definición de inclusión, considerando los aportes de distintas disciplinas. Específicamente, implicaría un desarrollo reflexivo sobre el área multidisciplinaria de la educación inclusiva. Como se puede observar al inicio de este artículo, existen distintas formas de entender la inclusión y al no evidenciarse una convergencia en aspectos básicos que sustentarían este constructo, se tiende a utilizar un discurso derivado de una tradición médica y, por tanto, muy acotado y referido a la educación especial. Sin embargo, una concepción contemporánea de la educación inclusiva hace referencia a todos los estudiantes y no sólo a aquellos que se les asigna la etiqueta de necesidades educativas especiales y discapacidad (Ainscow, 2005; Tregaskis, 2006). Por lo tanto, los futuros docentes necesitarían dejar atrás una visión de la diferencia con una orientación de déficit. El primer paso es trabajar la posibilidad de ver la inclusión como una totalidad, más que un área segregada a una especialidad (educación especial o interculturalidad). Siguiendo esta premisa, Ferri (2006) destaca la necesidad de animar a los estudiantes a que analicen cómo la clase social o la raza, por ejemplo, influye en cómo se vive la experiencia de una discapacidad. Más específicamente, este autor argumenta la importancia de que tanto los cursos de formación como sus respectivos materiales no destaquen características específicas de identidad (ej., un alumno con síndrome de Down evidencia alteraciones del habla) ya que esto negaría "la posibilidad de intersecciones entre o diferencias al interior de las categorías" (Ferri, 2006: 294). En síntesis, este planteamiento multidisciplinario demandaría una formación explícita y sustantiva en todas las formas de exclusión educacional existentes y el contexto social, cultural y político que permite que ésta se mantenga y reproduzca en el sistema escolar.

Finalmente, un último aspecto considera la necesidad de, como plantea Ainscow (2005), proporcionar nuevas formas de abordar la información utilizada para evaluar desempeño escolar. Al igual que las metodologías de enseñanza tradicionalmente se han centrado en el alumno promedio, también lo han hecho las formas en que se evalúan los aprendizajes. La medición de logros en la escuela ha sido muy rígida en contraste con la variabilidad de estudiantes que habitan una sala de clase contemporánea. Entonces, más que configurarse en una forma de potenciar el aprendizaje se ha constituido en un obstáculo a este. En este sentido, Ainscow sugiere que los educadores en formación desarrollen en sus cursos habilidades que les permitan construir estándares de inclusión que, por ejemplo, consideren procesos organizacionales de las escuelas y el uso de las visiones de los actores que habitan estos espacios.

En relación a los procesos organizacionales, la utilización de perspectivas teóricas más contemporáneas como los estudios culturales se constituye en un aporte a la formación docente (Slee, 2000). La formación de docentes que consideren el componente cultural como un constructo dinámico y producto de una construcción social compartida permitirá 
contextualizar las formas de funcionamiento que tiene cada comunidad educativa particular y abrirá posibilidades de gestionar acciones de cambio que faciliten el aprendizaje de todos los sujetos que participan de ella. Así, la inclusión educativa funcionaría no como una forma de identificar y reproducir la anormalidad sino como una herramienta de diagnóstico y desarrollo cultural (Ainscow, 2001). Por otro lado, la consideración y utilización de las miradas de los actores que participan de los establecimientos escolares potenciaría el desarrollo de estándares de inclusión educativa. Existe evidencia suficiente para respaldar la idea de que las escuelas deben ser construidas cooperativamente entre todos sus actores (Booth y Ainscow, 2000; Freire, 1998). Sin embargo, los sujetos que participan de esta inclusión no han sido escuchados respecto a sus deseos, experiencias y visiones sobre su aprendizaje y educación. La mayoría de la investigación contemporánea sobre las voces de los sujetos destaca el poder que tienen las metodologías de investigación y las formas de evaluación en enmarcar voces particulares, elicitando algunas y no otras (Thomson y Gunter, 2007). Esta mirada acepta la noción de que no existe una sola voz legitimada sino variedad de ellas, internamente diferenciadas por espacios, tiempos, relaciones y lugares (Lather, 1991). Desde esta perspectiva, los centros de formación docente debieran facilitar a sus estudiantes de herramientas que les permitiesen desarrollar nuevas formas de recoger y utilizar información, considerando a cada uno de los actores del sistema educativo.

\section{CONCLUSION}

En la actualidad se observan cambios significativos en la configuración de los establecimientos educacionales y los participantes de estos. Un gran número de sujetos que habían sido tradicionalmente excluidos del sistema educacional regular han ingresado a éste, dejando atrás la idea de que nuestras salas de clase estaban constituidas por un grupo homogéneo de alumnos en cuanto a sus habilidades, raza, género, lenguaje, estructura familiar, entre otros marcadores de identidades. Por lo tanto, esta nueva configuración de las instituciones educacionales implica una diversidad en los sujetos que las habitan. Una de las respuestas a esta diversidad ha sido un llamado desde organismos internacionales y estamentos gubernamentales a transformar las escuelas en comunidades más inclusivas. Sin embargo, a pesar del énfasis de la inclusión educativa en los discursos nacionales e internacionales, este concepto no tiene una significación única, facilitando y reproduciendo así las formas de exclusión que busca eliminar.

Así como otros conceptos contemporáneos que circulan en los discursos públicos (ej., interculturalidad), la inclusión educativa tiene sus orígenes en una tradición ligada a la educación especial y que proviene de una visión positivista de la realidad. Lo anterior tiene una serie de efectos al abordar el concepto de diversidad en el aprendizaje y la enseñanza de los sujetos, legitimando el concepto de normalidad como centro y paso a seguir. Es de esta forma como discursos de diagnóstico, categorización, asimilación y compensación se posicionan en las prácticas educativas, restringiendo el acceso de todos los sujetos a todos los espacios de aprendizaje y el acceso a una educación de calidad.

Debido a lo planteado anteriormente, la formación de profesores y profesoras se constituye en un desafío para las instituciones de educación superior y una forma de resig- 
nificar el concepto de inclusión. Al respecto, es necesaria la formación de un profesional que lidere las acciones educativas relacionadas con la diversidad desde la inclusión. Este sujeto no sólo debe concentrarse en la elaboración de herramientas técnicas que le permitan eliminar las barreras de acceso y participación de ciertos estudiantes a la educación sino analizar críticamente los propios sistemas de inclusión/exclusión y las representaciones y supuestos culturales adscritos a los diferentes marcadores de la subjetividad como por ejemplo, condición socioeconómica, formas de aprendizaje, nacionalidad, etnia, género, entre otros, que pueden repercutir en acciones de discriminación y opresión social. Del mismo modo, requiere que la formación en inclusión educativa considere los aportes de otras disciplinas y no sólo de la educación especial. La diversidad existente en cuanto a participantes del proceso educativo implica considerar el aprendizaje y la enseñanza desde distintas miradas que abordan ámbitos sociales y culturales más amplios y que requieren de una formación interdisciplinar. Finalmente, la formación docente requiere un mirar contextualizado del fenómeno educativo, donde la producción y uso del conocimiento es delimitada por posiciones geográficas, históricas y culturales del sujeto.

\section{BIBLIOGRAFIA}

Ainscow, M. (2001). Hacia escuelas eficaces para todos. Manual para la formación de equipos, España: Narcea.

Ainscow, M. (2005). Developing inclusive education systems: what are the levers for change. Journal of Educational Change, 6: 109-124.

Baker, B. (2002). The hunt for disability: The new eugenics and the normalization of school children. Teachers College Record, 104: 663-703.

Beyer, L.E. (2001). The value of critical perspectives in teacher education. Journal of Teacher Education, 52: 151-161.

Booth, T. y M. Ainscow (2000). Guía para la evaluación y mejora de la ecuación inclusiva. Index for inclusion, Madrid: Consorcio Universitario para la Educación Inclusiva.

Erevelles, N. (2006). Deconstructing difference: doing disability studies in multicultural contexts. En S. Danforth y S. Gabel, Vital questions facing disability studies in education (pp. 347-362). NY, New York: Peter Lang.

Ferri, B. A. (2006). Teaching to trouble. En S. Danforth y S. Gabel, Vital questions facing disability studies in education (pp. 289-306). NY, New York: Peter Lang.

Freire, P. (1998). Pedagogía del oprimido, México: Siglo Veintiuno.

Fuchs, D., \& L. S. Fuchs (1994). Inclusive schools movement and the radicalization of special education reform, Exceptional Children, 60: 294-309.

Graham, L. (2006). Caught in the net: a Foucaultian interrogation of the incidental effects of limited notions of inclusion, International Journal of Inclusive Education, 10: 3-25.

Graham, L. y R. Slee (2005). Inclusion? Artículo presentado en la Asociación Australiana para Investigación en Educación. Conferencia Anual, noviembre, Sydney.

Hall, S. (1997). Representation: Cultural representations and signifying practices, Thounsand Oaks, CA: Sage.

Infante, M., P. Ortega, M. Rodríguez, C. Fonseca, C. Matus y V. Ramírez (2008). (Co)Construcción de los discursos sobre diversidad. Trabajo presentado en Primer Congreso Internacional La Universidad y la Atención a la Diversidad cultural. De la discriminación a la inclusión, noviembre, Santiago, Chile.

Infante, M. y C. Matus (2009). Policies and practices on diversity: Reimagining possibilities for new discourses, Disability and Society (en prensa). 
Irarrázabal, I. y M. A. Morandé (2007). Cultura mapuche: entre la pertenencia étnica y la integración social, Estudios Públicos, 105: 37-59.

Lather, P. (1991). Getting smart: Feminist research and pedagogy within/in the postmodern, New York: Routledge.

Lipsky, D. K. \& A. Gartner (1996). Inclusion, school restructuring, and the remaking of American society, Harvard Educational Review, 66: 762-796.

MINEDUC (2005). Política Nacional de Educación Especial: Nuestro compromiso con la diversidad. Recuperado el 15 de julio de 2007, de http://www.mineduc.cl/biblio/documento/200704252105370.POLiTICAEDUCESP.pdf.

MINEDUC (2007). Programa de Educación Intercultural Bilingüe 2007. Recuperado el 3 de agosto de 2007, de http://www.mineduc.cl/ biblio/documento/200708240835400.ProgramadeEducaciOn.ppt.

Mutua, K. \& R. Smith (2006). Disrupting normalcy and the practical concerns of classroom teachers. En S. Danforth y S. Gabel, Vital questions facing disability studies in education (pp. 122-132), NY, New York: Peter Lang.

Rice, N. (2006). Teacher education as a site of resistance. En S. Danforth y S. Gabel, Vital questions facing disability studies in education (pp. 17-31), NY, New York: Peter Lang.

Riehl, C. J. (2000). The Principal's Role in Creating Inclusive Schools for Diverse Students: A Review of Normative, Empirical, and Critical Literature on the Practice of Educational Administration, Review of Educational Research, 70: 55-81.

Rose, N. (1999). Governing the soul, London, England: Free Association Books.

Slee, R. (2000). Keynote speech: talking back to power. The politics of educational exclusion. International Special Education Congress 2000. Including the excluded. University of Manchester $24^{\text {th }}-28^{\text {th }}$ July.

Slee, R. (2001). Inclusion in practice: does practice make perfect?, Educational Review, 53: $113-123$

Tenorio, S. (2007). Las representaciones sociales de profesores básicos de las comunas de Nuñoa y Macul acerca de la integración escolar. Tesis para optar al grado de Doctor en Ciencias de la Educación. Pontificia Universidad Católica de Chile, Santiago, Chile.

Thomson, P. \& H. Gunter (2007). The Methodology of Students-as-Researchers: Valuing and using experience and expertise to develop methods, Discourse: studies in the cultural politics of education, 28: 327-342.

Tregaskis, C. (2006). Developing inclusive practice through connections between home, community and school. En S. Danforth y S. Gabel, Vital questions facing disability studies in education (pp. 201-235), NY, New York: Peter Lang.

UNESCO (2005). Guidelines for inclusion: Ensuring Access to Education for All, París: UNESCO. 



\section{FE DE ERRATAS}

La pertenencia institucional de la profesora Silvia Redon Pantoja, autora del artículo "Autoevaluación institucional y acreditación como aseguramiento de la calidad de la educación: Implicancias teóricas y prácticas", es la Escuela de Pedagogía de la Pontificia Universidad Católica de Valparaíso.

Las colaboraciones, suscripciones y correspondencia deben ser dirigidas a la Dirección de la revista: Oficina de Publicaciones, Facultad de Filosofía y Humanidades, Universidad Austral de Chile, Casilla 567, Fono/Fax: 5663221275 , Valdivia, Chile. E-mail: eped@uach.cl

Las suscripciones incluyen los gastos de envío:

Chile: cheque o vale vista por \$10.000. Otros países: US\$ 15 .

El canje debe ser enviado a Biblioteca Central, Universidad Austral de Chile, Correo 2, Valdivia 
